\title{
Crescimento de Schizodon Intermedius Garavello \& Britski (Ostheichthyes, Anostomidae) do Rio Tibagi (Sertanópolis, Paraná)
}

\author{
Mário Luis Orsi ${ }^{1}$ \\ Oscar Akio Shibatta ${ }^{1}$
}

\begin{abstract}
Growth of Schizodon intermedius Garavello \& Britski (Ostheichthyes, Anostomidae) from Tibagi river (Sertanópolis, Paraná). This work consists of age, growth, and circuli formation period determination and the relation of reproduction, temperature, and pluviosity on the growth of $S$. intermedius Garavello \& Britski, 1990. The growth curve was adjusted by the Bertalanfy's expression and the relationship between weight and size was made by the minimum square method to determine the conditioning factor. Gonadossomatic index (IGS) was used to study the reproduction. The assintotic values for length were $33,94 \mathrm{~cm}$ and $44,81 \mathrm{~cm}$ for males and females, respectively. The circulus were formed annually and the growth was slow and the maximum age was determined to be nine years. The formation of the circuli was influenced by the reproduction, probably allied to other abiotic factors like temperature and rain.

KEY WORDS. Schizodon intermedius, age, growth, Tibagi river
\end{abstract}

Caracterizar a estrutura das populações de peixes é importante, pois gera algumas respostas sobre sua ecologia (VAZZOLER \& AMADIO 1990). Estudos visando o crescimento de uma população fornecem indicativos qualitativos do desenvolvimento da espécie, uma vez que o indivíduo ou a população revelam em seu crescimento as condições ambientais, bióticas e abióticas vigentes (BENEDITOCecílio \& AgostinHo 1997).

A piava-quatro-pintas, ou Schizodon intermedius Garavello \& Britski, 1990, um anostomídeo de médio porte, morfologicamente semelhante a Schizodon borelli (Boulenger, 1900), com a qual pode ser confundida, está distribuída na bacia do Alto Paraná, onde se inclui o rio Tibagi. Ela foi a sétima espécie mais abundante neste rio e a segunda mais capturada à sua jusante, no município de Sertanópolis. Provavelmente todo o ciclo de vida desenvolve-se nesta área e, do pouco que se conhece a respeito de seus hábitos, sabe-se que são herbívoras (BENNEMANN et al. 1995).

A maior comunidade de pescadores do rio Tibagi também está localizada no mesmo local; onde foi observado que $S$. intermedius ocupava um lugar de destaque na pesca comercial. Em recentes coletas nesta mesma região, verificou-se que a população de piava-quatro-pintas sofreu acentuado declínio.

1) Departamento de Biologia Animal e Vegetal, Centro de Ciências Biológicas, Universidade Estadual de Londrina. Caixa Postal 6001, 86051-970 Londrina, Paraná, Brasil.

E-mail: orsi@npd.uel.br 
Portanto, urgem pesquisas sobre a espécie. Tendo em vista a inexistência de trabalhos sobre sua dinâmica de crescimento, foram analisados os padrões de crescimento e etário, tanto quanto a relação de fatores como temperatura, pluviosidade e reprodução com o processo de desenvolvimento. Como comenta RICKER (1971), tais dados são importantes para a obtenção de informações sobre a composição dos estoques e ciclo de vida dos organismos.

\section{MATERIAL E MÉTODOS}

O trecho estudado no rio Tibagi ( $23^{\circ} 01^{\prime} 28^{\prime \prime S}$ e $\left.50^{\circ} 57^{\prime} 07^{\prime \prime W}\right)$, situa-se no município de Sertanópolis, próximo à foz do rio na região de planície final, norte do Paraná (MAACK 1981). Possui águas semi-lênticas e inúmeras baías, pois sofre influência da represa de Capivara (rio Paranapanema). A largura é de aproximadamente 500 metros e a profundidade pode atingir $25 \mathrm{~m}$. Para maiores detalhes ver BENNEMANN et al. $(1995,1996)$.

A amostra totalizou 295 exemplares de S. intermedius (102 machos e 193 fêmeas) coletados mensalmente, no período de julho de 1992 a julho de 1993. Para as capturas foram utilizadas redes de emalhar com 2 a $10 \mathrm{~cm}$ entre nós opostos.

De cada exemplar foram obtidos o comprimento padrão (Ls) em centímetros, peso total (Wt) em gramas, sexo e peso das gônadas. Na determinação do crescimento em comprimento, utilizou-se o método dos anéis etários em escamas, segundo VAZZOLER (1981). As escamas, em média seis, foram retiradas da região abaixo da nadadeira dorsal e acima da linha lateral, em seguida eram limpas, selecionadas e colocadas em $\mathrm{KOH}$ a $5 \% \mathrm{com}$ timol. Posteriormente eram secas e colocadas entre duas lâminas fixadas com fita adesiva. A leitura dos anéis etários foi feita em lupa estereoscópica (Nikon) em aumento de 10x.

Para o estudo da época de formação dos anéis utilizou-se a determinação gráfica entre o comprimento padrão médio bimestral e a classe de número de anéis. Para elaborar as curvas de crescimento em comprimento, para machos e fêmeas, foram utilizadas a transformação de Ford-Walford (WALFORD 1946) e a expressão de BERTALANFFy (1938), de acordo com SANTOS (1978) e VAZZOlER (1981).

Os dados de comprimento padrão e peso total foram transformados em logarítimos naturais e ajustados pelo método dos mínimos quadrados. O valor de b (ou $\theta$, constante relacionada com o tipo de crescimento dos indivíduos) foi estimado utilizando-se da equação $y=a \cdot x^{b}$ para ambos os sexos, para obter o fator de condição médio mensal com a equação $\mathrm{K}=\mathrm{Wt} / \mathrm{Lt}^{\theta}$. Para extrair a influência da reprodução no fator de condição, foi calculado também o fator de condição somático, K1, subtraindo-se o peso das gônadas do peso total. Os valores dos fatores de condição médio mensal, foram então lançados em gráfico para verificação a variação bimestral das condições de bem estar da espécie durante o período estudado.

Para a identificação dos sexos e análise do período reprodutivo através do índice gonadossomático médio bimestral (IGS), foram seguidos critérios de VAZZOLER $(1981,1996)$.

As informações referentes a temperaturas e pluviosidades médias bimestrais, foram obtidas dos relatórios mensais da EMATER, Paraná, regional de Sertanópolis, no período de julho de 1992 a julho de 1993. 


\section{RESULTADOS}

Os períodos de formação dos anéis etários, foram semelhantes para machos e fềmeas como pode ser observado através dos números de anéis por comprimentos médios bimestrais (Fig. 1A,B). Isto indica que a formação do anel etário é anual, ocorrendo entre os bimestres de dezembro a janeiro e de fevereiro a março, para o período estudado. Escamas com até oito anéis foram encontradas, para ambos os sexos, com uma única exceção, onde uma fềmea apresentou nove anéis etários. Esta foi excluída da análise, devido à raridade do evento, pois em coletas anteriores e posteriores ao presente estudo, nenhum indivíduo excedia este número de anéis. Acredita-se que oito anéis etários para a população em estudo dificilmente sejam ultrapassadas. Sendo que a idade calculada para oito anéis foi de 8,98 anos ou seja, aproximadamente nove anos.
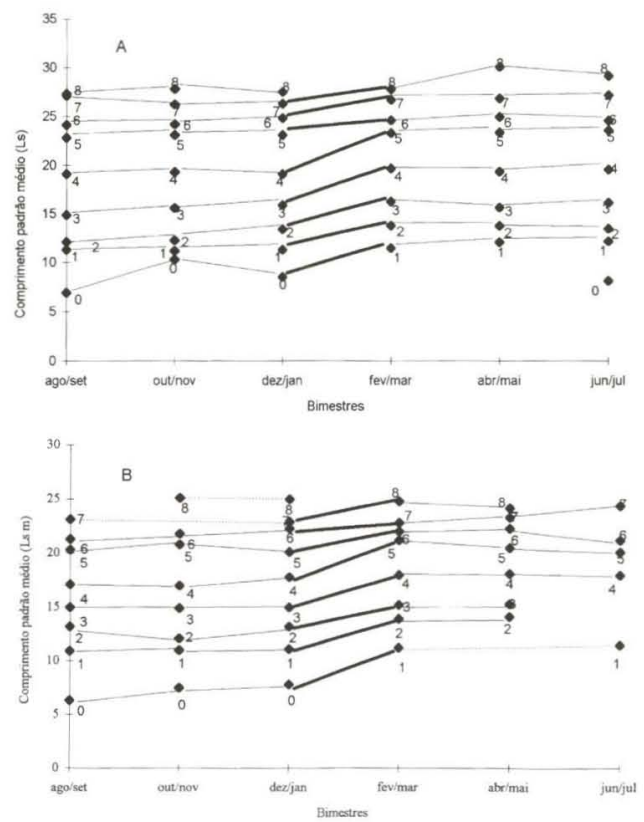

Fig. 1. Comprimento padrão médio bimestral (Ls) por classe de número de anéis para fêmeas (A) e machos (B) de Schizodon intermedius, durante o periodo de agosto/1992 a julho/1993.

O comprimento padrão médio e número de anéis etários relacionados, obtidos para ambos os sexos, estão apresentados na tabela I. Para machos e fêmeas, a variação do comprimento padrão mínimo e máximo observado foi de $6,3 \mathrm{~cm}$ a $33,2 \mathrm{~cm}$ e de $6,8 \mathrm{~cm}$ a $39,2 \mathrm{~cm}$ respectivamente. Foi evidenciado também que classes de três, quatro e cinco anéis etários foram mais freqüentes, para ambos os sexos, sendo encontrado o maior número de indivíduos com três anéis entre as fêmeas.

As relações lineares da transformação de Ford-Walford $\mathrm{L}_{\mathrm{s}}$ e $\mathrm{L}_{\mathrm{s}}+\Delta_{\mathrm{t}}$ para machos $(\mathrm{r}=0,98)$ e fêmeas $(\mathrm{r}=0,98)$ mostraram-se válidas à expressão de von Bertalanffy (Fig. 2A,B). Os valores de L $\infty$ e K (taxa de crescimento) obtidos para 
machos foram 33,94 cm e 0,1018. Já para as fêmeas foram de 44,81 cm e 0,1262, respectivamente. Para ambos os sexos consideramos $\mathrm{T}_{0}$ assumindo o valor zero, pois o valor foi desprezível se comparado ao valor médio máximo que a espécie pode atingir. Assim, chegamos às seguintes expressões matemáticas de von Bertalanffy: $\mathrm{Ls}=33,94\left(1-\mathrm{e}^{-0,1018^{*} \mathrm{~T}}\right)$ para machos; e $\mathrm{Ls}=44,81\left(1-\mathrm{e}^{-0,1262 * \mathrm{~T}}\right)$ para fêmeas.

Tabela I. Dados sobre comprimento padrão médio obtido por classe de idade e por sexo, para Schizodon intermedius.

\begin{tabular}{cccc}
\hline \multirow{2}{*}{ Anéis etários } & $\begin{array}{c}\text { Idade } \\
\text { (anos) }\end{array}$ & \multicolumn{2}{c}{ Comprimento padrão médio $(\mathrm{cm})$} \\
\cline { 3 - 4 } & & Machos & Fêmeas \\
\hline 0 & 0,00 & 0,00 & 0,00 \\
1 & 1,98 & 6,14 & 7,80 \\
2 & 2,98 & 10,67 & 13,26 \\
3 & 3,98 & 13,43 & 17,36 \\
4 & 4,98 & 15,86 & 21,07 \\
5 & 5,98 & 18,08 & 24,43 \\
6 & 6,98 & 19,9 & 27,46 \\
7 & 7,98 & 21,56 & 30,19 \\
8 & 8,98 & 23,03 & 32,66 \\
\hline
\end{tabular}
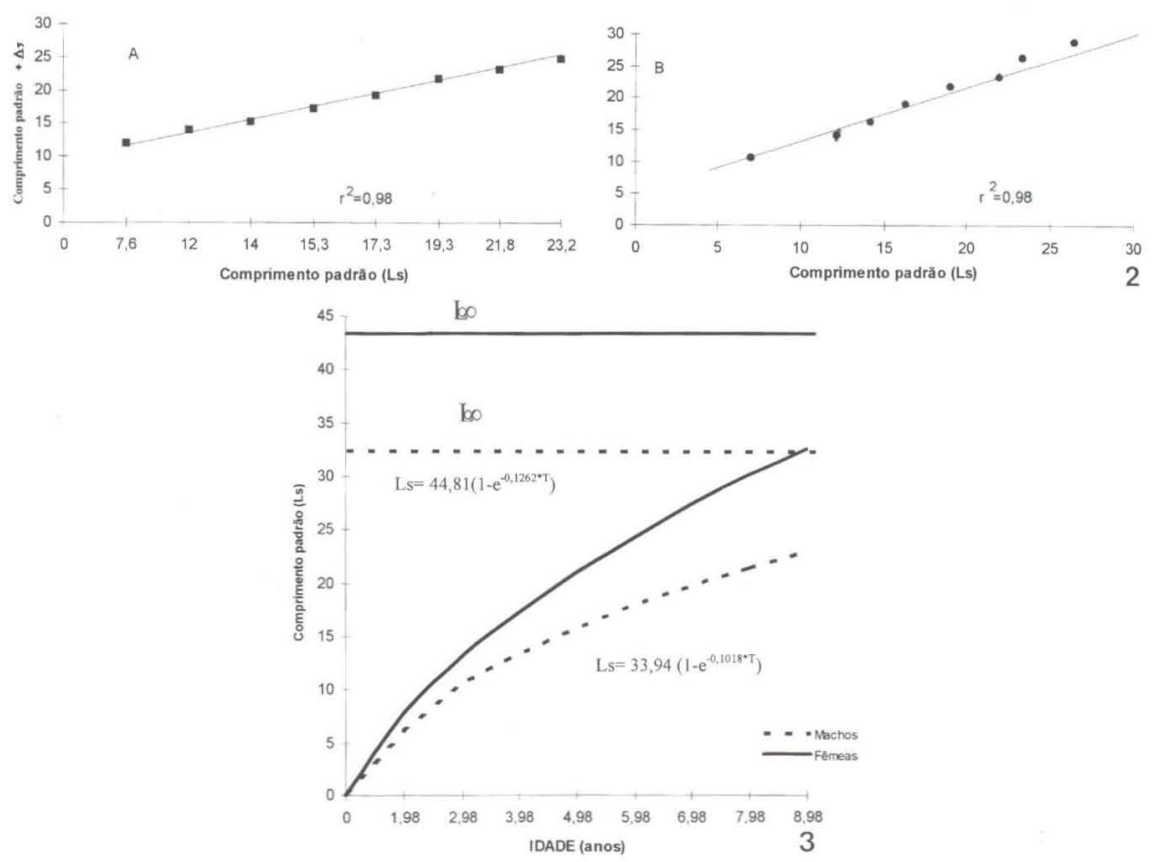

Figs 2-3. Schizodon intermedius. (2) Relação Ford-Walford para machos (A) e fêmeas (B) no período de agosto/92 a julho/93; (3) curva de crescimento em comprimento pelo método de Bertalanffy de machos e fêmeas. 
O gráfico das curvas de crescimento em comprimento para machos e fêmeas encontram-se na figura 3, demonstrando um padrão de crescimento lento para ambos os sexos. Onde as fêmeas atingem maior valor assintótico e taxa de crescimento.

Os dados obtidos para o índice gonadossomático mostram valores elevados no bimestre de dezembro-janeiro, indicando ser o provável período de reprodução da espécie estudada. Apenas uma moda foi observada, evidenciando desova total. Cabe ressaltar que esta tendência coincide com os valores elevados da temperatura média bimestral como demonstrado nas figura 4A,B. Apesar da reprodução ter ocorrido quando a pluviosidade (Fig. 4C) apresentava-se elevada, as relações com a pluviosidade são mais sutis, necessitando investigações mais detalhadas. A pluviosidade ocorre em épocas de maiores temperaturas, porém o seu valor máximo não coincidiu com aqueles de temperatura e reprodução.
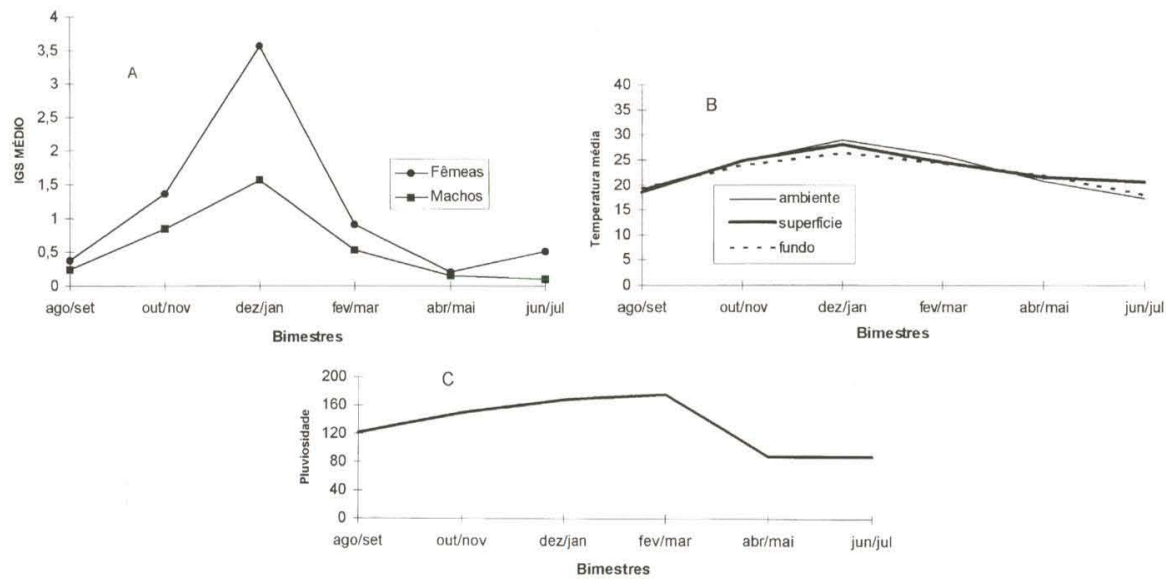

Fig. 4. (A) Índice gonadossomático médio bimestral para machos e fêmeas de Schizodon intermedius, temperaturas (B) e Pluviosidade (C) bimestrais no período de agosto/92 a julho/93.

$\mathrm{O}$ valor estimado para $\theta$ da relação peso/comprimento (Fig. 5A,B) em machos e fêmeas foi respectivamente de 2,60 e 3,27. Ou seja, indicando um crescimento alométrico positivo com incremento do peso em relação ao comprimento para as fêmeas, mas não em machos, onde o crescimento é alometricamente negativo. Estes valores, portanto, conferem dimorfismo sexual para a espécie.

Quanto ao fator de condição K e K1 (Fig. 6A,B ), observou-se que ocorreram variações temporais em ambos os sexos, com acréscimo dos valores no bimestre de dezembro - janeiro, o que coincide com aqueles valores elevados do índice gonadossomático e temperatura. Entretanto, um teste " $\mathrm{t}$ " entre $\mathrm{K}$ e $\mathrm{K} 1$ de ambos os sexos evidenciou que as diferenças em machos não são significativas $(t=1,37$, nível de significância $=0,183, \alpha=0,05)$ mas sim em fêmeas $(\mathrm{t}=2,48$, nível de significância $=0,016, \alpha=0,05)$. Assim, verificou-se que o fator de condição K1 demonstra que as fêmeas demandam um valor considerável de energia para a formação das gônadas no período reprodutivo. 

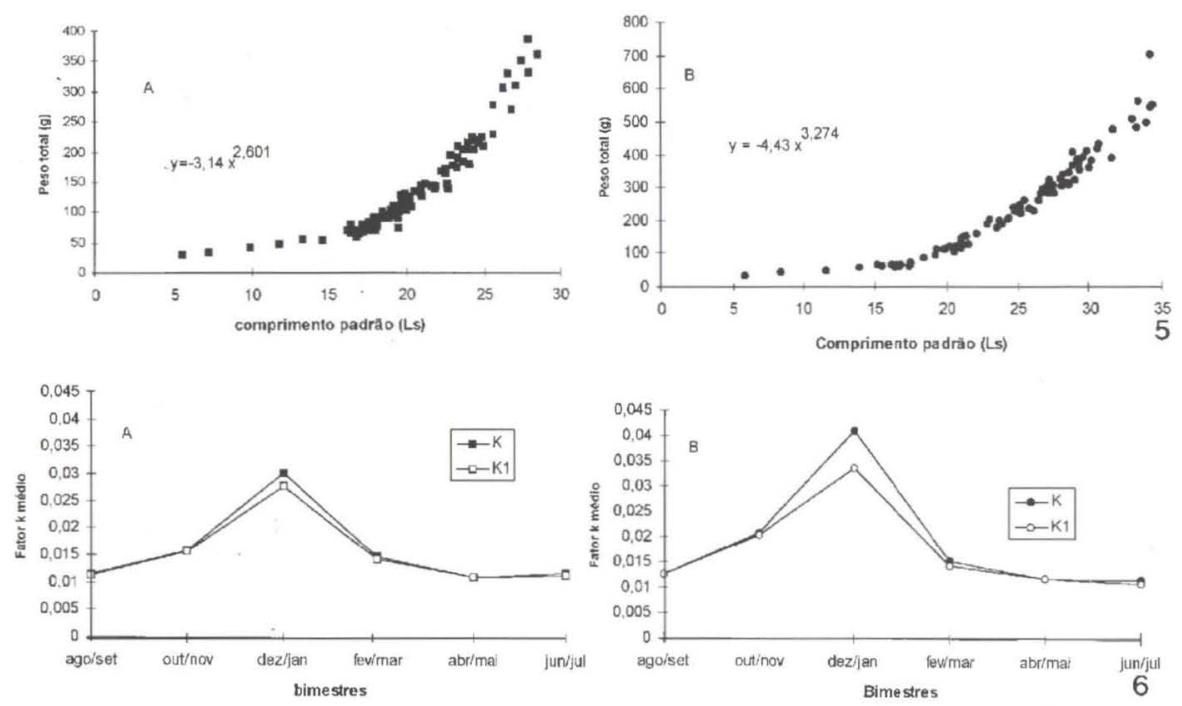

Figs 5-6. Schizodon intermedius. (5) Relação peso (g)/ comprimento padrão (cm) para machos (A) e fêmeas (B) no periodo de agosto/92 a julho/93; (6) valores médios do fator de condição $k$ e k1 (excluido as gônadas) de machos $(A)$ e fêmeas $(B)$.

\section{DISCUSSÃO}

Durante o período estudado, Schizodon intermedius pôde ser considerada uma espécie residente pois esteve presente em todos os meses. Através da proporção sexual, nota-se que existem mais fêmeas do que machos na população. Além disso, poucos indivíduos com idades avançadas foram capturados na região e ficou evidente que o crescimento da espécie é lento.

O crescimento em peixes não apresenta um caráter uniforme (MENON 1953; SANTOS 1978; BARBIERI \& BARBIERI 1983; GURGEL 1995), podendo ser acelerado em determinadas épocas, lento e até nulo em outras. Essas variações são evidenciadas em algumas estruturas duras do corpo do animal, através de marcas concêntricas, denominadas anéis etários, os quais representam períodos de crescimento lento ou nulo. Por isso, as escamas foram empregadas neste estudo. A validade da utilização das escamas nos estudos sobre crescimento tem sido discutida para os peixes neotropicais, uma vez que nos trópicos as variações ambientais tais como temperatura e luminosidade não são muito marcantes como nas regiões de clima temperado. Porém, confirma-se que o método também pode ser aplicado para Schizodon intermedius, onde ainda ficou evidente que a temperatura deve ser um fator relevante na reprodução da espécie. Outros trabalhos de pesquisadores brasileiros demonstram que essa metodologia também poderá ser aplicada satisfatoriamente aos nossos peixes (SANTOS \& BARBIERI 1992).

As escamas retiradas da região abaixo da nadadeira dorsal e acima da linha lateral de Schizodon intermedius, mostraram mais claramente os anéis, como observado por GIAMAS et al. (1995) em Schizodon nasutus Kner, 1858. BLAKE \& 
BlAKE (1978, apud SANTOS \& BARBIERI 1992) enfatizam que nos trópicos a formação de anéis pode ser influenciada por vários fatores bióticos e abióticos, os quais muitas vezes variam a intensidade e sincronia de ano para ano. Como para outras espécies (SANTOS \& BARBIEIRI 1992; BARBIERI \& BARBIEIRI 1983), a maturação gonadal parece ter sido um dos processos mais importantes na formação dos anéis etários em $S$. intermedius, estando a época de reprodução diretamente associada a este acontecimento

O hábito alimentar, a disponibilidade de alimento e a fisiologia própria da espécie (OLMSTED \& KILAMBI 1978), poderiam também ocasionar o aparecimento de anéis etários em peixes. Poucos são os estudos no Brasil que contrastam o suposto padrão de crescimento mais lento em peixes herbívoros, como $S$. intermedius, com peixes de outros regimes alimentares. Porém, OKEYO (1989) salienta que peixes estritamente herbívoros e de crescimento mais lento necessitam de proteína animal como complemento para adquirir um crescimento mais rápido e pleno. Em estudos sobre alimentação, YABE \& BENNEMANN (1994) demonstraram que $S$. intermedius possui um hábito alimentar preferencialmente herbívoro, alimentando-se das macrófitas ribeirinhas. BENNEMANN et al. (1996) em estudos com a mesma espécie e no mesmo período do presente estudo, mostraram que a maior atividade alimentar ocorre nos meses de verão, logo após o esforço reprodutivo, onde também as reservas de gordura cavitária tornam-se menores. Portanto, o incremento alimentar através de aporte de proteína animal não foi verificado nesta espécie. Provavelmente porque o alimento não foi um fator limitante ao crescimento, pois a disponibilidade deste ocorreu durante todo o ano, com variações apenas na quantidade de macrófitas no ambiente.

A periodicidade da formação do anel etário em $S$. intermedius foi semelhante a resultados obtidos por SANTOS \& BARBIERI (1992) para outro anostomídeo, Leporinus piau Fowler, 1941, onde o aparecimento também foi anual. Coincidiu também com o máximo de elevação e declínio do período reprodutivo, conforme curvas do índice gonadossomático médio bimestral (IGS).

Quanto à taxa de crescimento das fêmeas observa-se o contrário em $S$. intermedius daqueles estudos realizados por BARBIERI \& SANTOS (1988) para Leporinus friderici (Bloch, 1794) e SANTOS \& BARBIERI (1992) para Leporinus piau, em que as fêmeas possuem taxas de crescimento menores do que os machos.

Para as diferenças encontradas nos $\mathrm{L} \infty$ entre os sexos, muitos autores sugerem que a reprodução pode ser a principal causa, e que isso pode ser conseqüência da dimensão e manutenção das gônadas. VAZZOLER (1996) sugere além do fator reprodução, aspectos comportamentais e ambientais, ligados à reprodução, que afetam tanto o comprimento como o tamanho de primeira maturação para os peixes.

A diferença do tamanho assintótico entre os sexos observada em $S$. intermedius não deve ser um evento raro na família. O mesmo foi encontrado em $S$. knerii Steindachner, 1875 (SATO \& BARBIERI 1983) e L. piau, onde as fêmeas ultrapassaram os machos a partir de determinada idade (SANTOS \& BARBIERI 1992). Além da taxa de crescimento diferenciada, VAZZOLER (1996) observa um maior tamanho de corpo geralmente em fêmeas de espécies que não cuidam da prole, como característica sexual secundária. 
A variação do fator de condição estimado por nós para $S$. intermedius, demonstrou estar associado ao período de desova para esta espécie, como já observado em estudo de BARBIERI \& VERANI (1987). Este fato confirma as observações de LE CREN (1958), onde o fator de condição, por se tratar de um parâmetro relacionado a fisiologia do peixe, pode variar segundo a concentração de gordura, variação de parâmetros ambientais, condições alimentares, idade e desenvolvimento gonadal.

Segundo VAZZOLER (1996) a reprodução e o crescimento são processos de produção e competem pelos mesmos recursos limitados. Para $S$. intermedius foi evidenciado que a alocação de recursos energéticos pareceu ser máxima para a reprodução, acarretando assim uma interrupção do crescimento somático. Estes eventos podem ser corroborados pela alta correlação do período de formação dos anéis etários com o período reprodutivo. Segundo Fontoura \& Agostinho (1996), outros fatores, como por exemplo a elevação de temperatura, pluviosidade e fotoperíodo, também devem ser considerados pois influenciam todo um processo fisiológico. Ficou evidente que $S$. intermedius pode ter sofrido interferências da temperatura e pluviosidade na reprodução e, conseqüentemente, no crescimento.

A partir da teoria das espécies com estratégias reprodutivas $\mathrm{r}, \mathrm{K}$ e sazonal, algumas caraterísticas obtidas de $S$. intermedius, o enquadram como estrategista sazonal. O padrão apresentado por esta espécie é comparável ao descrito por WINEMILLER (1989), que considera animais de estratégia sazonal um grupo intermediário entre os estrategistas $\mathrm{r}$ e K. Desta forma, o tamanho do corpo varia de intermediário a grande, ocorrem grandes flutuações em populações locais, os ovócitos são pequenos, o período reprodutivo é curto e o investimento de recursos energéticos para a reprodução é grande na época chuvosa.

Assim, o crescimento de Schizodon intermedius segue um padrão semelhante para a família Anostomidae, porém com ritmo mais lento, que determina um porte médio. Este processo pode ser decorrente do hábito e regime alimentar, bem como a forma de utilização dos recursos no habitat.

\section{CONCLUSÕES}

O crescimento em machos e fêmeas de $S$. intermedius é realizado de forma lenta e alométrica, o que pode ser caracterizado pelo seu hábito e regime alimentar, sendo muito influenciado pela reprodução e condições ambientais vigentes no período.

Ambos os sexos podem apresentar idades de até nove anos, sendo simples a visualização pelos anéis etários nas escamas .

Os valores do comprimento assintótico médio $(\mathrm{L} \infty)$ para a espécie são maiores nas fêmeas do que nos machos, o mesmo com a taxa de crescimento.

Devido às características de crescimento e algumas táticas de reprodução evidenciadas, tais como a ausência de cuidado parental e período reprodutivo curto, a espécie necessita de um acompanhamento e estudos mais aprofundados, pois pode tornar-se vulnerável a agressões antrópicas em seu ambiente. 
AGRADECIMENTOS. Ȧ Dra. Sirlei T. Bennemann e Ângela T.S. Souza que coordenaram o trabalho de campo no período de estudo. Ao técnico Edson Santana da Silva, pelo trabalho de coleta e toda equipe de coleta de peixes do projeto Tibagi. A Heitor Frossard pelo auxílio na redação. Ao Sr. Manoel Reis que emprestou sua propriedade rural às margens do rio Tibagi. $\mathrm{O}$ presente estudo foi financiado pelo convênio UEL/COPATI/KLABIN.

\section{REFERÊNCIAS BIBLIOGRÁFICAS}

BARBIERI, G. \& M.C. BARBIERI. 1983. Growth and first sexual maturation size of Gymnotus carapo Linnaeus, 1758 in the Lobo reservoir (State of São Paulo, Brazil) (Pisces, Gymnotidae). Rev. Hydrobiol. Trop. 16 (2): 195-201.

BARBIERI, G. \& E.P. SANTOS. 1988. Ánalise comparativa do crescimento e de aspectos reprodutivos da piava, Leporinus friderici (Bloch, 1794) (Osteichthyes, Anostomidae) da represa do Lobo e do Rio Mogi-Guaçu, Estado de São Paulo. Ciência e Cultura, São Paulo, 40 (7): 693-697.

BARBIERI, G. \& J.R. VERANI. 1987. O fator de condição como indicador do período de desova em Hypostomus aff. plecostomus (Linnaeus, 1758) Osteichthyes, Loricariidae), na represa do Monjolinho (São Carlos, SP). Ciência e Cultura, São Paulo, 39 (7): 655-658.

Benedito-Cecílio, E. \& A.A. Agostinho. 1997. Estrutura das populações de peixes do reservatório de segredo, p.113-139. In: A.A. AgostinHo \& L.C. GOMES (Ed.). Reservatório de segredo: bases ecológicas para o manejo. Maringá, Eduem, 387p.

Bennemann, S.T., A.T. Silva-Souza \& G.R.A. Rocha. 1995. Composicion ictiofaunistica en cinco localidades de la cuenca del Rio Tibagi, PR - Brasil. Interciencia 20 (1): 7-13.

Bennemann, S.T.; M.L. Orsi \& O.A. Shibatta. 1996. Atividade alimentar de espécies de peixes do rio Tibagi, relacionada com o desenvolvimento de gordura e das gônadas. Revta bras. Zool. 13 (2): 501-512.

BERTALANFFY, L. VON. 1938. A quantitative theory of organic growth. Hum. biol. 10 (2): $181-213$.

FonTOURA, N.F. \& A.A. AgostinHo. 1996. Growth with seasonally varying, temperatures: an expansion of the Von Bertalanffy growth model. Jour. Fish Biol. 48: 569-584.

Giamas, M.T.D.; R.A. SAnTos; H. Vermulm JR.; E.C. CAMPos \& J.J.C. DA CAMARA. 1995. Estimativa da curva de crescimento, através da lepidologia, relacionada com o tamanho de primeira maturação gonadal do Ximborê Schizodon nasutus Kner, 1859 (Ostheichthyes, Anostomidade) na represa de Ibitinga, (21ํ6'S-4859'W), Estado de São Paulo, Brasil. Bol. Inst. Pesca, São Paulo, 22 (2): 103-110.

GURGEL, H. DE C.B. 1995. Estrutura da população de Metynnis cf. roosevelti Eigenmann, 1915 (Characidae, Myleinae), da lagoa Redonda, Nízia Floresta, Rio Grande do Norte - Brasil. Bol. Inst. Pesca, São Paulo, 22 (1): 61-71.

LE CREN, E.D. 1958. Observations on the growth of perch (Perca fluviatilis L.) over twenty-two years with special reference to the effects of temperature and changes in populations density. Jour. Anim. Ecol. 27 (2): 287-334. 
MAACK, R. 1981. Geografia Física do Estado do Paraná. Rio de Janeiro, José Olympio Editora, 450p.

MENON, M.D. 1953. The determination of age and growth of fishes in tropical and sub-tropical waters. Jour. Bombay Nat. Hist. Soc. 51 (3): 623-635.

OKEYO, D.O. 1989. Herbivory in freshwater fishes: A review. Israel Jour. Aquacult., Bamidgeh, 41 (3): 79-97.

Omlsted, L.L. \& R.V. Kilambi. 1978. Age and growth of spotted bass (Micropterus punctulatus)in Lake Fort Smith, Arkansas. Trans. Amer. Fish. Soc. 107 (1): $21-25$.

RICKER, W.E. 1971. Methods for assessment of fish production in fresh waters. Oxford, Blackeell Scientific Publications, 348p.

SANTOS, E.P. DOS. 1978. Dinâmica de populações aplicada à pesca e piscicultura. São Paulo, Hucitec/Edusp, 129p.

SAntos, G.B. \& G. BARBIERI. 1992. Idade e crescimento do Piau Gordura, Leporinus piau Fowler, 1941, na represa de Três Marias (Estado de Minas Gerais) (Pisces, Ostariophysi, Anostomidae). Rev. Brasil. Biol. 53 (4): 649-658.

SAto, Y \& G. BARBIERI. 1983. Crescimento de Schizodon knerii Steindachner, 1875 (Pisces, Anostomidae) na represa de Três Marias, MG. An. Sem. Reg. Ecol., São Carlos, 3: 201-221.

VAzzoler, A.E.A. DE M. 1981. Manual de métodos para estudos biológicos sobre populações de peixes. Crescimento e reprodução. Brasília, CNPq, Programa Nacional de Zoologia, 108p.

1996. Biologia da reprodução de peixes teleósteos: Teoria e Prática. Maringá, Sociedade Brasileira de Ictiologia, 169p.

VAzzoler, A.E.A. DE M \& S.A. AMAdio. 1990. Aspectos biológicos de peixes amazônicos. XIII. Estrutura e comportamento de cardumes multiespecíficos de Semaprochilodus (Characiformes, Prochilodontidae) do baixo rio Negro, Amazonas, Brasil. Rev. Brasil. Biol. 50 (3): 537-546.

WALFORD, L.A. 1946. A new graphic method of describing the growth of animals. Biol. Bull. Mar. Biol. Lab. Woods Hole 90: 141-147.

WinEMILLER, K.O. 1989. Patterns of variation in life history among South American fishes in seasonal environmentals. Oecologia 81: 225-241.

YABE, R.S. \& S.T. BENNEMANN. 1994. Regime alimentar de Schizodon intermedius Garavello \& Britski do rio Tibagi, Paraná, sua relação com algumas características morfológicas do trato digestivo (Ostheichthyes, Anostomidae). Revta bras. Zool. 11 (4): 777-788.

Recebido em 13.I.1998; aceito em 06.VI.1999. 